\title{
The Serum 25(OH)D Level in Patients with Atypical Femoral Fracture
}

\author{
Hiroyuki Tsuchie $^{1 *}$, Naohisa Miyakoshi ${ }^{1}$, Yuji Kasukawa ${ }^{1}$, Toshiaki Aizawa $^{2}$, Hidekazu Abe ${ }^{3}$, \\ Toyohito Segawa ${ }^{3}$, Yoichi Shimada ${ }^{1}$
}

\author{
${ }^{1}$ Department of Orthopedic Surgery, Akita University Graduate School of Medicine, Akita, Japan \\ ${ }^{2}$ Department of Orthopedic Surgery, Northern Akita Municipal Hospital, Kitaakita, Japan \\ ${ }^{3}$ Ugo Municipal Hospital, Ugo, Japan \\ Email: *tuchikiti@yahoo.co.jp
}

How to cite this paper: Tsuchie, H., Miyakoshi, N., Kasukawa, Y., Aizawa, T., Abe, H., Segawa, T. and Shimada, Y. (2018) The Serum 25(OH)D Level in Patients with Atypical Femoral Fracture. Open Journal of Orthopedics, 8, 331-336.

https://doi.org/10.4236/ojo.2018.89036

Received: August 9, 2018

Accepted: September 8, 2018

Published: September 11, 2018

Copyright (C) 2018 by authors and Scientific Research Publishing Inc. This work is licensed under the Creative Commons Attribution International License (CC BY 4.0)

http://creativecommons.org/licenses/by/4.0/

\begin{abstract}
Objective: Excessive curvature of the femur has been considered to be one of the factors associated with atypical femoral fracture (AFF). Although we clarified that femoral curvature was markedly influenced by a low vitamin $\mathrm{D}$ level, very few studies have been conducted on the direct relationship between AFF and a serum low level of vitamin D. Therefore, we compared the serum vitamin D level between AFF and osteoporosis patients. Methods: A total of 48 consecutive AFFs in 36 Japanese patients were retrospectively reviewed using the medical records, and 12 consecutive AFFs in 9 patients (AFF group) whose 25-hydroxyvitamin D $(25(\mathrm{OH}) \mathrm{D})$ levels were examined were included in this study. As a non-AFF control group, 45 consecutive age-matched female patients with osteoporosis (Osteoporosis group) were enrolled. We compared some laboratory examination items, the bone mineral density, and curvature of the femur. Results: When univariate logistic regression analysis was performed to compare the 2 groups, Ca levels and lateral and anterior curvatures in the AFF group were significantly greater than in the Osteoporosis group $(\mathrm{P}<0.05, \mathrm{P}<0.01$, and $\mathrm{P}<0.01$, respectively), and the $25(\mathrm{OH}) \mathrm{D}$ level in the AFF group was significantly lower than in the Osteoporosis group $(\mathrm{P}<0.01)$. We used discriminant analysis of these extracted items to exclude the mutual influence of factors and identify factors closely associated with $\mathrm{AFF}$, and $25(\mathrm{OH}) \mathrm{D}, \mathrm{Ca}$ and anterior curvature were identified as significant items to differentiate these diseases $(\mathrm{P}<0.05, \mathrm{P}<0.01$, and $\mathrm{P}<$ 0.01, respectively). Conclusion: AFF patients showed low vitamin D levels and marked femoral curvatures.
\end{abstract}

\section{Keywords}

Fracture, 25(OH)D, Curvature, Femur 


\section{Introduction}

Although bisphosphonates (BPs) are some of the main drugs for osteoporosis patients, several side effects due to their long-term use have been reported in recent years, such as atypical low-energy subtrochanteric and diaphyseal femoral fractures due to markedly suppressed bone turnover (SSBT) [1] [2]. These fractures are typically diagnosed as atypical femoral fracture (AFF). However, many patients have been reported with AFF without BP therapy [3], and multiple factors are thought to affect AFF development. In recent years, excessive femoral curvature has been considered to be one of the factors associated with AFF [4] [5]. Because the pathogenesis of femoral curvature in elderly women is unknown, we investigated whether several factors related to bone metabolism and posture affect the development of femoral curvature in elderly women. As a result of this study, we clarified that femoral curvature was strongly influenced by a low vitamin D level [6]. In addition, we previously reported a case of osteomalacia with excessive curvature of the femur, being consistent with the definition of AFF [7]. We need to understand the direct relationship between AFF and a low serum level of vitamin $\mathrm{D}$ when we consider these results. However, very few studies have been conducted on this relationship.

The aim of this study was to compare the serum vitamin D level between AFF and osteoporosis patients.

\section{Methods}

\subsection{Subjects}

A total of 48 consecutive AFFs in 36 Japanese patients who visited our institutions between January 2006 and December 2017 were retrospectively reviewed using their medical records, and 12 consecutive AFFs in 9 patients (AFF group), with a mean age of 79.9 years (59 to 90 ), whose 25-hydroxyvitamin D (25(OH)D) levels were examined at the first visit were finally included in this study. Three patients showed bilateral AFFs, and the remaining 6 patients showed unilateral AFFs. As a non-AFF control group, 45 consecutive age-matched female patients with osteoporosis (Osteoporosis group) and without diabetes mellitus or glucocorticoid usage who visited our institutions between September 2016 and December 2017 were enrolled. In the AFF group, 8 patients ( 9 fractures) had been prescribed osteoporosis treatments prior to evaluation: bisphosphonate, vitamin $\mathrm{D}_{3}$, teriparatide, denosumab, and selective estrogen receptor modulator, were prescribed to $1,4,1,1$, and 1 patient, respectively. In the Osteoporosis group, 23 patients had received osteoporosis treatments prior to evaluation: bisphosphonate, vitamin $\mathrm{D}_{3}$, teriparatide, denosumab, and selective estrogen receptor modulator, were prescribed to $12,7,2,2$, and 4 patients, respectively.

\subsection{Clinical Evaluations}

We performed some laboratory examinations of vitamin D metabolism markers 
and bone metabolic markers: 25-hydroxyvitamin D (25(OH)D), alkaline phosphatase (ALP), calcium ( $\mathrm{Ca}$ ) adjusted for serum albumin, inorganic phosphorus (IP), intact procollagen I N-terminal propeptide (P1NP), tartarate-resistant acid phosphatase 5b (TRACP5b), and intact parathyroid hormone (PTH). We measured the bone mineral density (BMD) on AP views of the lumbar spine from $\mathrm{L} 2$ to L4 and the femoral neck (Discovery, Hologic Inc., MA, USA). Curvature of the femur was measured with antero-posterior (A-P) and lateral radiographs, using a method to measure femoral curvature described by Yau et al. [8]. The diaphysis of the femur was divided into four equal parts and a line was then drawn in each quarter to highlight the midpoint of the endosteal canal. The degree of femoral curvature was defined as the angle formed between the central line of the proximal femoral diaphysis and central line of the distal femoral diaphysis. We measured the unaffected femur in the complete AFF patients and the affected femur in the incomplete AFF patients. In the bilateral incomplete AFF patients, we measured both femurs and used the average of the two values.

\subsection{Statistical Analysis}

All values are expressed as the mean \pm standard deviation (SD). Student's $t$-test and the Welch $t$-test were used to compare the AFF and Osteoporosis groups. Discriminant analysis was used to examine effective factors for distinguishing the two diseases. Probability $(\mathrm{P})$ values less than 0.05 were considered significant.

\section{Results}

All of the AFF patients were female and had diaphyseal fractures. Five AFFs were complete fractures, and the remaining 7 AFFs were incomplete fractures. All complete AFFs and 5 incomplete AFFs were treated surgically with intramedullary nail fixation or a locking plate. Intramedullary nail fixation was used for 6 AFFs, and a locking plate was used for 4 AFFs. There were no diabetes mellitus patients or patients receiving glucocorticoids (Table 1).

As a result of univariate logistic regression between the 2 groups, Ca levels and lateral and anterior curvatures in the AFF group were significantly greater than in the Osteoporosis group, and the $25(\mathrm{OH}) \mathrm{D}$ level in the AFF group was significantly lower than in the Osteoporosis group. We used discriminant analysis of these extracted items to exclude the mutual influence of factors and identify factors closely associated with $\mathrm{AFF}$, and $25(\mathrm{OH}) \mathrm{D}, \mathrm{Ca}$, and anterior curvature were identified as significant items to differentiate these diseases (Table 2).

\section{Discussion}

In this study, we examined serum $25(\mathrm{OH}) \mathrm{D}$ and some parameters in AFF patients, and showed that the $25(\mathrm{OH}) \mathrm{D}$ level in the AFF patients was lower than in the osteoporosis patients and femoral curvature was more marked than in the osteoporosis patients. Although only one study investigating 20 AFF patients 
Table 1. Clinical information on patients with AFF.

\begin{tabular}{cc}
\hline Variables & AFF patients \\
Numbers & 9 \\
Female/Male & $9 / 0$ \\
Unilateral/Bilateral & $6 / 3$ \\
Right/Left & $8 / 4$ \\
Diaphyseal/Subtrochanteric & $12 / 0$ \\
Complete/Incomplete & $5 / 7$ \\
Surgical treatment & 10 \\
Intramedullary nail/Locking plate & $6 / 4$ \\
Presence of diabetes mellitus & 0 \\
Glucocorticoid usage & 0 \\
\hline
\end{tabular}

AFF: atypical femoral fracture.

Table 2. Univariate logistic regression and discriminant analyses to compare 2 groups.

\begin{tabular}{|c|c|c|c|c|}
\hline & & & $\begin{array}{l}\text { Student's } t \text {-test } \\
\text { Welch } t \text {-test }\end{array}$ & $\begin{array}{c}\text { Discriminant } \\
\text { analysis }\end{array}$ \\
\hline Variables & Osteoporosis & $\mathrm{AFF}$ & $P$ & $P$ \\
\hline Number & 45 & 9 & & \\
\hline Age (years) & $80.1 \pm 10.0$ & $79.9 \pm 10.3$ & 0.962 & \\
\hline \multicolumn{5}{|l|}{ Laboratory examinations } \\
\hline ALP (IU/L) & $248 \pm 104$ & $248 \pm 103$ & 0.993 & \\
\hline $\mathrm{Ca}(\mathrm{mg} / \mathrm{dL})$ & $9.14 \pm 0.38$ & $9.64 \pm 0.62$ & 0.043 & $<0.001$ \\
\hline IP (mg/dL) & $3.49 \pm 0.42$ & $3.61 \pm 0.69$ & 0.619 & \\
\hline P1NP $(\mu g / L)$ & $58.9 \pm 37.2$ & $44.5 \pm 22.4$ & 0.296 & \\
\hline TRACP5b (mU/dL) & $285 \pm 138$ & $389 \pm 267$ & 0.316 & \\
\hline Intact PTH (pg/mL) & $42.6 \pm 22.1$ & $37.0 \pm 18.6$ & 0.504 & \\
\hline $25(\mathrm{OH}) \mathrm{D}(\mathrm{ng} / \mathrm{mL})$ & $23.7 \pm 8.5$ & $13.3 \pm 3.5$ & $<0.001$ & 0.030 \\
\hline \multicolumn{5}{|l|}{$\operatorname{BMD}\left(\mathrm{g} / \mathrm{cm}^{2}\right)$} \\
\hline Lumbar spine & $0.719 \pm 0.155$ & $0.717 \pm 0.189$ & 0.961 & \\
\hline Proximal femur & $0.470 \pm 0.118$ & $0.512 \pm 0.218$ & 0.632 & \\
\hline \multicolumn{5}{|l|}{ Femoral curvature (angle) } \\
\hline Lateral & $3.4 \pm 3.5$ & $12.1 \pm 5.5$ & $<0.001$ & 0.968 \\
\hline Anterior & $10.5 \pm 2.5$ & $18.4 \pm 5.0$ & 0.002 & $<0.001$ \\
\hline
\end{tabular}

Values are expressed as number of patients or mean \pm SD with ranges. AFF: atypical femoral fracture, ALP: alkaline phosphatase, Ca: calcium, IP: inorganic phosphorus, P1NP: intact procollagen I N-terminal propeptide, TRACP5b: tartarate-resistant acid phosphatase 5b, PTH: intact parathyroid hormone, 25(OH)D: 25-hydroxyvitamin D, BMD: bone mineral density. 
reported a relationship between AFF and a low serum 25(OH)D level, this was not described in detail [9]. There have been no reports on the relationship between AFF and serum 25(OH)D since this study. Although marked femoral curvature in AFF patients is not a novel finding, a low level of vitamin D in AFF patients has been scarcely reported. Considering that femoral curvature was strongly influenced by a low vitamin $\mathrm{D}$ level, as the next step, we must examine the association between femoral curvature and serum 25(OH)D solely in AFF patients. In order to clarify this in the future, we need to analyze more AFF patients, considering the use of a bone resorption inhibitor.

In general, osteomalacia patients often show a low serum Ca level. On the contrary, serum $\mathrm{Ca}$ in AFF patients was higher than in osteoporosis patients. Because the mechanism of the relationship between low serum 25(OH)D and high $\mathrm{Ca}$ is not clear, many AFF patients and evaluation items must be investigated in the future.

A limitation of this study was the small number of AFF patients. Several factors may have led to a bias, such as subtrochanteric or diaphyseal, and the presence or absence of bisphosphonate administration, and a larger number of patients is needed to sufficiently analyze these factors. As another limitation, detailed clinical information, such as bone turnover markers, bone mineral density, and other contributing medical conditions before fractures, was not available for many patients because most were referred from other clinics or hospitals on an emergency basis. AFF is a relatively rare fracture, and more patient information must be accumulated to clarify the influence of these factors.

\section{Conclusion}

To the best of our knowledge, the present study is the first to compare the serum vitamin D level between AFF and osteoporosis patients, and AFF patients showed low vitamin D levels. We need to perform further detailed studies with more AFF patients to clarify the influence of vitamin D on such patients.

\section{Conflicts of Interest}

The authors declare no conflicts of interest regarding the publication of this paper.

\section{References}

[1] Bamias, A., Terpos, E. and Dimopoulos, M.A. (2010) Avascular Osteonecrosis of the Jaw as a Side Effect of Bisphosphonate Treatment. Onkologie, 33, 288-289. https://doi.org/10.1159/000313684

[2] Odvina, C.V., Zerwekh, J.E., Rao, D.S., Maalouf, N., Gottschalk, F.A. and Pak, C.Y. (2005) Severely Suppressed Bone Turnover: A Potential Complication of Alendronate Therapy. The Journal of Clinical Endocrinology \& Metabolism, 90, 1294-1301. https://doi.org/10.1210/jc.2004-0952

[3] Tan, S.C., Koh, S.B.J., Goh, S.K. and Howe, T.S. (2010) Atypical Femoral Stress Fractures in Bisphosphonate-Free Patients. Osteoporosis International, 22, 2211-2222. 
https://doi.org/10.1007/s00198-010-1384-1

[4] Sasaki, S., Miyakoshi, N., Hongo, M., Kasukawa, Y. and Shimada, Y. (2012) Low-Energy Diaphyseal Femoral Fractures Associated with Bisphosphonate Use and Severe Curved Femur: A Case Series. Journal of Bone and Mineral Metabolism, 30, 561-567. https://doi.org/10.1007/s00774-012-0358-0

[5] Shin, W.C., Moon, N.H., Jang, J.H., Park, K.Y. and Suh, K.T. (2017) Anterolateral Femoral Bowing and Loss of Thigh Muscle Are Associated with Occurrence of Atypical Femoral Fracture: Effect of Failed Tension Band Mechanism in Mid-Thigh. Journal of Orthopaedic Science, 22, 99-104. https://doi.org/10.1016/j.jos.2016.09.009

[6] Tsuchie, H., Miyakoshi, N., Kasukawa, Y., Senma, S., Narita, Y., Miyamoto, S., Hatakeyama, Y., Sasaki, K. and Shimada, Y. (2016) Factors Related to Curved Femur in Elderly Japanese Women. Upsala Journal of Medical Sciences, 121, 170-173.

https://doi.org/10.1080/03009734.2016.1185200

[7] Tsuchie, H., Miyakoshi, N., Nishi, T., Abe, H., Segawa, T. and Shimada, Y. (2015) A Case of Incomplete Atypical Femoral Fracture with Histomorphometrical Evidence of Osteomalacia. Acta Medica Okayama, 69, 59-63.

[8] Yau, W.P., Chiu, K.Y., Tang, W.M. and Ng, T.P. (2007) Coronal Bowing of the Femur and Tibia in Chinese: Its Incidence and Effects on Total Knee Arthroplasty Planning. Journal of Orthopaedic Surgery (Hong Kong), 15, 32-36. https://doi.org/10.1177/230949900701500108

[9] Girgis, C.M., Sher, D. and Seubel, M.J. (2010) Atypical Femoral Fractures and Bisphosphonate Use. The New England Journal of Medicine, 362, 1084. https://doi.org/10.1056/NEJMc0910389 